\title{
Mapping viscoelastic and plastic properties of polymers and polymer-nanotube composites using instrumented indentation
}

\author{
Andrew J. Gayle and Robert F. Cook ${ }^{\text {a) }}$ \\ Materials Measurement Science Division, National Institute of Standards and Technology, \\ Gaithersburg, MD 20899, USA
}

(Received 26 February 2016; accepted 12 May 2016)

\begin{abstract}
An instrumented indentation method is developed for generating maps of time-dependent viscoelastic and time-independent plastic properties of polymeric materials. The method is based on a pyramidal indentation model consisting of two quadratic viscoelastic Kelvin-like elements and a quadratic plastic element in series. Closed-form solutions for indentation displacement under constant load and constant loading-rate are developed and used to determine and validate material properties. Model parameters are determined by point measurements on common monolithic polymers. Mapping is demonstrated on an epoxy-ceramic interface and on two composite materials consisting of epoxy matrices containing multiwall carbon nanotubes. A fast viscoelastic deformation process in the epoxy was unaffected by the inclusion of the nanotubes, whereas a slow viscoelastic process was significantly impeded, as was the plastic deformation. Mapping revealed considerable spatial heterogeneity in the slow viscoelastic and plastic responses in the composites, particularly in the material with a greater fraction of nanotubes.
\end{abstract}

\section{INTRODUCTION}

Indentation of polymeric or biological materials or their composites frequently leads to three concurrent modes of contact deformation, characterized by three different variations of the indenter displacement into the material, $h$, with indentation load, $P$, and time, $t^{1-12}$ :

(V) Viscous deformation, in which the indenter displacement is time-dependent, typically with the rate of displacement varying with load.

(E) Elastic deformation, in which the indenter displacement is time-independent and recovers completely on load removal.

(P) Plastic deformation, in which the indenter displacement is time-independent and does not recover at all on load removal.

Indentation with a spherical probe or flat punch often suppresses plastic deformation such that the deformation is completely viscoelastic, ${ }^{6,11,13-20}$ or is assumed so for fluid-like materials. ${ }^{14,15,21,22}$ Under these conditions, viscoelastic correspondence principles ${ }^{23-27}$ can be used to predict the $h(t)$ response from an imposed $P(t)$ spectrum (or vice versa) if the purely elastic $P(h)$ indentation behavior is known and a time-dependent creep (or relaxation) function is selected for the material. The predictions are frequently framed in terms of superposition integrals. Indentation with the more-commonly used

Contributing Editor: Erik G. Herbert

a) Address all correspondence to this author.

e-mail: robert.cook@nist.gov

DOI: $10.1557 /$ jmr.2016.207 pyramidal probes, such as the three-sided Berkovich diamond, usually generates all three of the viscouselastic-plastic (VEP) indentation deformations. Under these conditions, correspondence principles, which rely on material linearity in elastic and viscous constitutive behavior (Hookean and Newtonian, respectively), cannot be used as the plasticity renders the nonviscous component of the deformation nonlinear. Hence, although correspondence methods can incorporate the geometrical nonlinearity of spherical and pyramidal indentation, in which the indentation contact area increases with indentation depth, ${ }^{25}$ plastic deformation precludes their use.

The original VEP indentation model incorporated the geometrical nonlinearity of pyramidal indentation explicitly into each component of the deformation, ${ }^{1}$ treating each displacement component separately. The plastic displacement, $h_{\mathrm{P}}(t)$, was given by

$$
h_{\mathrm{P}}(t)=\left[P_{\max }(t) / \alpha_{1} H\right]^{1 / 2},
$$

where $P_{\max }(t)$ is the maximum load experienced over the time interval $t, \alpha_{1}$ is a dimensionless indenter geometry constant, and $H$ is the resistance to plastic deformation. For an elastic-perfectly plastic material $H$ is the hardness. The elastic displacement, $h_{\mathrm{E}}(t)$, was given by

$$
h_{\mathrm{E}}(t)=\left[P(t) / \alpha_{2} M\right]^{1 / 2},
$$

where $\alpha_{2}$ is another dimensionless indenter geometry constant, and $M$ is the resistance to elastic deformation. For an elastic material, $M$ is the indentation modulus. 
The viscous displacement, $h_{\mathrm{V}}(t)$, was expressed as a rate,

$$
\mathrm{d} h_{\mathrm{V}}(t) / \mathrm{d} t=\left[P(t) / \alpha_{2} M \tau^{2}\right]^{1 / 2},
$$

where $\tau$ is the time constant for viscous flow. The term $\alpha_{2} M \tau^{2}$ is an effective quadratic viscosity. [Eqs. (2) and (3) use the more recent notation. $\left.{ }^{4}\right]$ The total indentation displacement was taken as the sum

$$
h=h_{\mathrm{E}}+h_{\mathrm{P}}+h_{\mathrm{V}}
$$

such that the overall indentation model could be viewed as a generalized quadratic "Maxwell"-like model of elements in series. For simple loading schemes, such as triangular, linear load-unload, spectra, the integral implicit in Eq. (3) is simply performed and the total displacement given by Eq. (4) can be expressed in closed form. The simplicity of this formulation allows the resistance to the three different modes of deformation to be scaled separately via $H, M$, and $\tau$, such that the complete variety of material indentation load-displacement responses can be described and analyzed. ${ }^{4,6}$

The quadratic Maxwell VEP model above was reasonably successful in a quantitative sense: the material properties $H, M$, and $\tau$ inferred from fits of the model to triangular load spectra, specifically fits to the unloading response in which all three displacement components are distinct, were in agreement with other measurements ${ }^{1,2,5}$; the ability of the model to predict loading behavior from unloading behavior was excellent ${ }^{1,2,4,5,8}$; and the material properties inferred using a triangular load spectrum at a reference time- or load-scale could be used to predict the triangular responses for indentation time scales factors of three shorter or longer than the reference and indention load-scales factors of ten different from the references. ${ }^{1,2,4,8}$ The limitations of the quadratic Maxwell VEP model are apparent in comparisons of observed and predicted behavior during constant-load creep, a test method that focuses on the time-dependent constitutive behavior. The free viscous element, meaning that it has no element constraining it in parallel, of the quadratic Maxwell VEP model leads to unbounded displacement increasing linearly with time for an imposed constant load and an instantaneous displacement recovery on load removal. ${ }^{1}$ Neither of these phenomena are observed during indentation of the vast majority of polymeric and biological materials: Displacement rates during constant-load creep decrease with time such that the displacement approaches a bounded value at long times $1,3,7,9,11,12,20$ and recovery on load removal is not instantaneous but occurs over time scales comparable to those required to reach the bounded creep displacement. ${ }^{9,12}$ Furthermore, the time-dependent indentation creep and recovery behavior of most polymeric and biological materials is not well described by a singletime-constant, but by two or more. , $^{3,12,16,18-20}$

Here, a new VEP pyramidal indentation model and analysis is developed that is similar in spirit to the original model (elements in series, closed-form displacement expressions, simple identification of material behavior dependencies) but which overcomes the deficiencies noted above. As foreshadowed earlier, ${ }^{4}$ the model is based on a quadratic "Kelvin"-like element, in which the viscous element is bounded by an elastic element in parallel, such that displacement during constant-load creep is bounded and displacement recovery on load removal is not instantaneous. Two such viscoelastic elements are combined in series to provide for deformation over two time scales, along with a third, plastic, element in series as before. A schematic diagram of the full quadratic Kelvin VEP model is shown in Fig. 1. The model is nearly identical to that recently implemented by Mazeran et al. ${ }^{9}$ and Isaza et al., ${ }^{12}$ with the omission of the fourth quadratic Maxwell element used by Mazeran, Isaza et al. to describe a viscoplastic response. In the original VEP model, the behavior of the singletime-constant viscous element could be deconvoluted unambiguously from the indentation unloading response of a simple load triangle. The extra degree of freedom associated with the two time constants of the two viscoelastic elements in Fig. 1 precludes such simple deconvolution and a more extensive testing protocol is

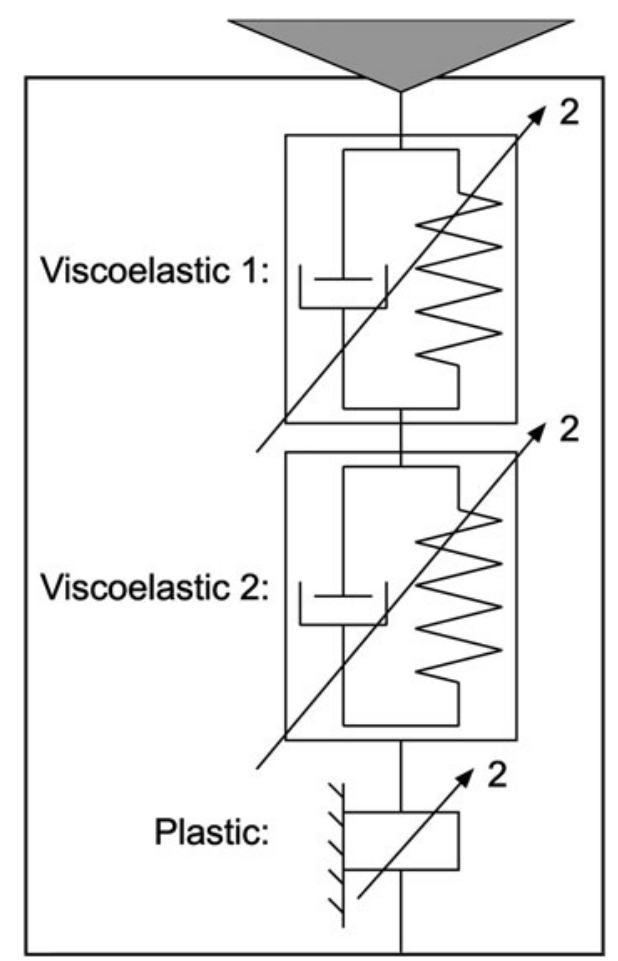

FIG. 1. Schematic diagram of the pyramidal indentation quadratic Kelvin VEP model. The model consists of two quadratic viscoelastic Kelvin elements in series with a quadratic plastic element. 
required. Here, a three-segment load spectrum is used as shown in Fig. 2(a): (i) a triangle wave, followed by (ii) a near-zero load hold, followed by (iii) a trapezoidal load wave. The protocol is identical to that used by Zhang et al. ${ }^{3}$ for exactly the same reasons: the final trapezoidal segment isolates the viscoelastic response under constantload conditions that are simple to analyze.

A major motivation for the development of the new model and analysis was to map the mechanical properties of polymer-carbon nanotube (CNT) composites. Such composites have great potential as lightweight mechanical or electrical materials that take advantage of the high stiffness and strength or electrical conductivity of CNTs, respectively. As with all composites, appropriate dispersion of the minority enhancing phase in the majority matrix phase is critical to obtaining desired properties: For example, the stiff CNTs must be dispersed and bound to the compliant polymer matrix so as to achieve stress transfer and stiffening of the composite; the conducting CNTs must be dispersed so as to percolate throughout the insulating polymer matrix so as to obtain a conducting structure. As a consequence, considerations of CNT dispersion in polymer matrices have been the subject of much study since the early applications of CNTs. ${ }^{28-31}$

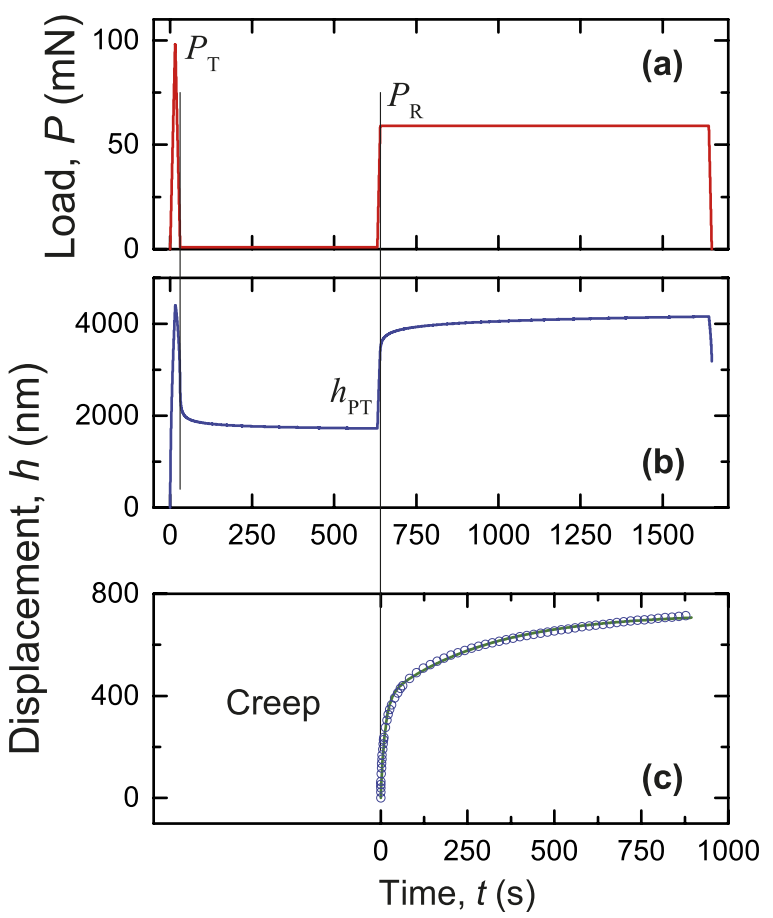

FIG. 2. Experimental load-displacement-time, $P-h-t$, data for the PMMA1 material during the three-segment indentation test. (a) The imposed triangle-hold-trapezoid load spectrum; the end of the initial triangle to peak load $P_{\mathrm{T}}$ and the beginning of the creep segment at load $P_{\mathrm{R}}$ of the final trapezoid are indicated by vertical lines. (b) The resulting displacement response; at the end of the central hold the displacement is $h_{\mathrm{PT}}$. (c) The displacement in the creep segment of the final trapezoid in shifted coordinates.
The goal here was to develop an instrumented indentation testing (IIT) method that could provide a direct measure of the mechanical behavior of polymer-CNT composites in the form of two-dimensional maps of viscoelastic and plastic properties. Such maps take advantage of the local probing capabilities of IIT and have been used to explore the effects of microstructure on spatial variations of mechanical properties in ceramic-metal composites,${ }^{32}$ tooth enamel, ${ }^{33}$ bone,${ }^{34}$ cement paste and rocks, ${ }^{35}$ and metals. ${ }^{36,37}$ In these cases, time-dependent deformation was not considered and elastic and plastic properties were mapped. The next section develops the new VEP indentation model and expressions for $h(t)$ that allow material properties to be determined from simple $P(t)$ protocols. The following sections then demonstrate and validate the applicability of the model in single-point tests on monolithic polymers and glass. The mapping capability is then demonstrated in multipoint tests on a ceramic-polymer interface and two polymer-CNT composites. Finally, possible extensions of the model and protocols are discussed.

\section{MATERIALS AND METHODS}

\section{A. Materials}

Five common commercial polymeric materials were obtained in approximately $1 \mathrm{~mm}$ thick solid sheet form. The materials were a high-density polyethylene (HDPE), two poly(methyl methacrylate)s (PMMA1, and PMMA2), a polystyrene (PS), and a polycarbonate (PC). Samples approximately $15 \mathrm{~mm} \times 15 \mathrm{~mm}$ were cut from the sheets and fixed to aluminum pucks for IIT measurements. The surfaces of the samples were highly reflective and tested in the as-received state, except for the HDPE, which was diamond polished to a reflective state. A commercial sodalime silicate glass (SLG) microscope slide was also tested.

Two commercial epoxy materials were obtained in liquid form as separate resins and curing agents. The first (Epoxy1) consisted of Epon 828 resin (miller-stephenson, Danbury, CT) and Ancamide 507 curing agent (Air Products, Allentown, PA). The resin and curing agent were mixed in the proportion 3:1 by mass and samples approximately $5 \mathrm{~mm}$ thick cast into circular plastic molds $35 \mathrm{~mm}$ in diameter, allowed to cure at room temperature for $48 \mathrm{~h}$, and then removed from the molds. The surfaces cast against the base of the molds were highly reflective and tested in the as-cast state. The second (Epoxy2) consisted of a resin and curing agent used for metallographic sample preparation, mixed as directed by the supplier (Buehler, Lake Bluff, IL), cast into a circular plastic mold along with a piece of dense aluminum nitride (AlN) ceramic, and allowed to cure as directed. The composite sample was removed from the mold and diamond polished to a reflective state such that a sharp epoxy-ceramic interface was normal to the sample surface. 
Two epoxy-CNT composite materials were obtained from a commercial vendor. The composites consisted of mass fractions of $1 \%$ and $5 \%$ of multiwall CNT (MWCNT) mixed into an epoxy matrix equivalent to Epoxy1 above. To enable direct comparison with the mechanical properties of the matrix epoxy, no chemical additives to improve homogeneity of MWCNT dispersion were included in the composites; it was thus expected that the MWCNT dispersion would be somewhat inhomogeneous. ${ }^{29,30}$ The composite material samples were in the form of $15 \mathrm{~mm} \times 15 \mathrm{~mm}$ sheets approximately $0.5 \mathrm{~mm}$ thick and were fixed to aluminum pucks with Epoxy1 for IIT measurements. The surfaces of the samples were reflective and tested in the as-received state.

\section{B. Experimental methods}

Single-point IIT measurements of the monolithic materials were used to establish and validate the experimental and analytical methods in two sequential stages. In the first stage, indentation displacements were measured during a three-segment applied load spectrum as described above. Material properties were determined from these measurements. In the second stage, indentation displacements were predicted from these properties for a range of single-segment linear load ramps and compared with experimental measurements; the property values were also compared with accepted values for the materials. In this way, the validity of both the form of the analysis and the magnitude of the included parameters could be assessed. A Berkovich diamond probe was used for all experiments. The data collection rate for load, displacement, and time for all experiments was at least $10 \mathrm{~Hz}$.

The first-stage, three-segment applied load spectrum was as follows:

(i) A triangular segment, consisting of a linear load ramp from zero to a peak load of $P_{\mathrm{T}}$ and then a linear ramp back to near zero over a total period of approximately $30 \mathrm{~s}$; the end of this segment is indicated by the left vertical line in Fig. 2. $P_{\mathrm{T}}=100 \mathrm{mN}$ was used. The displacement response for PMMA1 is shown in Fig. 2(b) and consisted of an increase from zero to a peak displacement followed by a decrease to a nonzero displacement at the end of the segment.

(ii) A period of near zero load, approximately $1 \mathrm{mN}$, typically extending for $600 \mathrm{~s}$. The displacement response for PMMA1 is shown in Fig. 2(b) and consisted of recovery to a near invariant displacement, $h_{\mathrm{PT}}$, at the end of the segment.

(iii) A trapezoidal segment consisting of a linear ramp from near zero load to peak load of $P_{\mathrm{R}}<P_{\mathrm{T}}$ in a time of $t_{\mathrm{R}}$, a long creep load-hold period at $P_{\mathrm{R}}$, and a linear ramp to zero load. $P_{\mathrm{R}}=60 \mathrm{mN}$ and $t_{\mathrm{R}} \approx 10 \mathrm{~s}$ were used and the creep hold period was typically $1000 \mathrm{~s}$; the beginning of the creep hold period is indicated by the right vertical line in Fig. 2. The displacement response for PMMA1 is shown in Fig. 2(b) and consisted of a rapid increase in displacement followed by a slower increase to near invariant displacement at the end of the creep hold. An expanded view of the slower creep displacement response is shown in shifted creep coordinates in Fig. 2(c) (setting the creep time to 0 after the $t_{\mathrm{R}} \approx 10 \mathrm{~s}$ rise to the creep load); analysis of such data provided the majority of the material viscoelastic information. At least four threesegment spectra were measured and analyzed for each material. Prior to measurement, preliminary trapezoidal experiments were used to ascertain the characteristic time scales for deformation.

The second-stage validation experiments were a series of linear load ramps. The ramps were part of a triangular load wave as illustrated in the initial segment of Fig. 1(a), with the exceptions that the peak loads were variable values $P_{\mathrm{U}}$ and the rise times were much longer variable values $t_{\mathrm{U}}$. (The subscript "U" indicates that these validation conditions are in principle unknown during the above first-stage measurements.) Only the loading parts of the triangle waves were used in the validation experiments. Two different forms of validation experiments were performed on PMMA1: (i) loading to a fixed peak load of $P_{\mathrm{U}}=100 \mathrm{mN}$ with variable rise times of $t_{\mathrm{U}}=(20,50,100,200,500,1000$, and 2000) s; and, (ii) loading with fixed rise time of $t_{\mathrm{U}}=500 \mathrm{~s}$ and variable peak loads of $P_{\mathrm{U}}=(20,50,100,200$, and 500$) \mathrm{mN}$. A single form of validation experiment was performed on the set of monolithic materials: loading with a fixed rise time of $t_{\mathrm{U}}=1000 \mathrm{~s}$ to a peak load of $P_{\mathrm{U}}=100 \mathrm{mN}$ or $P_{\mathrm{U}}=50 \mathrm{mN}$ (HDPE only).

Multipoint measurements of the epoxy-ceramic interface and the epoxy-CNT composites were used to generate line scans and maps of viscoelastic and plastic deformation properties. Linear arrays of 10 indentations on $100 \mu \mathrm{m}$ centers were performed over randomly selected areas of the composites for direct quantitative comparisons of properties with the base epoxy. A two-dimensional $10 \times 10$ array of indentations was performed over a $900 \mu \mathrm{m} \times 900 \mu \mathrm{m}$ square centered on the interface in the epoxy-ceramic sample to demonstrate the mapping capability in an extreme case of spatial property variation. Similar arrays of indentations were performed on randomly selected areas of the epoxy-CNT composites to demonstrate the mapping capability in inhomogeneous polymer microstructures. The three-segment test sequence described above was used for all the multipoint measurements; the total test time for the two-dimensional arrays was nearly $48 \mathrm{~h}$.

\section{Analysis method}

The analytical method developed here will be used to extract material mechanical properties from displacement measurements during the three-segment experimental applied load spectrum. The total indentation displacement 
at all times is given by the sum of the plastic and viscoelastic displacements, truncating Eq. (3) to

$$
h=h_{\mathrm{P}}+h_{\mathrm{VE}},
$$

where the viscoelastic displacement, $h_{\mathrm{VE}}$, is now given by the sum of the displacements of two quadratic Kelvin elements in series, Fig. 1,

$$
h_{\mathrm{VE}}=h_{\mathrm{VE} 1}+h_{\mathrm{VE} 2} .
$$

The plastic displacement is as before, Eq. (1). The displacement for each quadratic Kelvin element is described by a differential equation [see Appendix, Eq. (A4)],

$$
\frac{\mathrm{d} h_{\mathrm{VE} i}}{\mathrm{~d} t}+\frac{h_{\mathrm{VE} i}}{\tau_{i}}=\left(\frac{P(t)}{\alpha_{2} M_{i} \tau_{i}^{2}}\right)^{1 / 2},
$$

where $i=1$ or 2 , and (for each element) $M_{i}$ is the viscoelastic resistance, and $\tau_{i}$ is the time constant for viscoelastic deformation. [In the limit of $\tau_{i}>>\mathrm{d} t / \mathrm{dln}$ $h_{\mathrm{VE} i}$, Eq. (6) reverts to the form of Eq. (3).]

At the end of the triangular segment (i), the displacement consists of the sum of plastic deformation given by Eq. (1) and viscoelastic deformation given by an expression similar to Eqs. (A10a) and (A10b), but the relative proportions are not known. At the end of the recovery segment (ii), the viscoelastic deformation has decayed to near zero, if the segment is long enough, similar to Eq. (A11), such that only displacement associated with plastic deformation remains:

$$
h_{\mathrm{PT}}=\left[P_{\mathrm{T}} / \alpha_{1} H\right]^{1 / 2} .
$$

The "T" subscript in $h_{\mathrm{PT}}$ indicates that the plastic displacement is set by the maximum load attained, $P_{\mathrm{T}}$ (Fig. 2) and remains invariant thereafter. Equation (7) allows $\alpha_{1} H$ to be determined for the material from the measured values of $h_{\mathrm{PT}}$ and $P_{\mathrm{T}}$.

As $P_{\mathrm{R}}<P_{\mathrm{T}}$ in the trapezoidal segment (iii), there is no further plastic deformation and the additional displacement is completely viscoelastic and described by an expression similar to Eq. (A9). In particular, during the hold of the trapezoidal segment, the viscoelastic displacement is given by solving the differential equation for each quadratic Kelvin element for fixed load to gain

$$
\begin{aligned}
h_{\mathrm{VE}}=h_{\mathrm{R}} & +\left[\left(\frac{P_{\mathrm{R}}}{\alpha_{2} M_{1}}\right)^{1 / 2}-h_{\mathrm{R} 1}\right]\left[1-\exp \left(-\frac{\left(t-t_{\mathrm{R}}\right)}{\tau_{1}}\right)\right] \\
+ & {\left[\left(\frac{P_{\mathrm{R}}}{\alpha_{2} M_{2}}\right)^{1 / 2}-h_{\mathrm{R} 2}\right]\left[1-\exp \left(-\frac{\left(t-t_{\mathrm{R}}\right)}{\tau_{2}}\right)\right] . }
\end{aligned}
$$

$h_{\mathrm{R}}$ is the total displacement at the end of the ramp of the trapezoidal segment, and thus the displacement at the beginning of the hold segment; $h_{\mathrm{R} 1}$ and $h_{\mathrm{R} 2}$ are the contributions of each viscoelastic element to the total, $h_{\mathrm{R}}=h_{\mathrm{R} 1}+h_{\mathrm{R} 2}$. Fitting Eq. (8) to the measured $h_{\mathrm{VE}}(t)$ response enables the time constants $\tau_{1}$ and $\tau_{2}$ to be determined, along with the amplitudes characterizing each exponential term. An example fit for PMMA1 is shown in Fig. 2(c), using the natural creep coordinates from Eq. (8) of $t-t_{\mathrm{R}}$ and $h_{\mathrm{VE}}-h_{\mathrm{R}}$. During the ramp of the trapezoidal segment, the viscoelastic displacement is given by solving the differential equation for each quadratic Kelvin element for linearly increasing load, such that at peak ramp load, $P_{\mathrm{R}}$, the displacement $h_{\mathrm{R}}$ is given by

$$
\begin{aligned}
h_{\mathrm{R}}=\left(\frac{P_{\mathrm{R}}}{\alpha_{2} M_{1}}\right)^{1 / 2}\left[1-\frac{\sqrt{\pi}}{2}\left(\tau_{1} / t_{\mathrm{R}}\right)^{1 / 2} \exp \left(-t_{\mathrm{R}} / \tau_{1}\right) \operatorname{erf}\left(t_{\mathrm{R}} / \tau_{1}\right)^{1 / 2}\right] \\
+\left(\frac{P_{\mathrm{R}}}{\alpha_{2} M_{2}}\right)^{1 / 2}\left[1-\frac{\sqrt{\pi}}{2}\left(\tau_{2} / t_{\mathrm{R}}\right)^{1 / 2} \exp \left(-t_{\mathrm{R}} / \tau_{2}\right) \operatorname{erf}\left(t_{\mathrm{R}} / \tau_{2}\right)^{1 / 2}\right]
\end{aligned}
$$

Combining Eqs. (8) and (9) allows the contributions to the amplitude terms, $\alpha_{2} M_{1}$ and $\alpha_{2} M_{2}$ and $h_{\mathrm{R} 1}$ and $h_{\mathrm{R} 2}$, to be separated and determined for the material from the measured values of $h_{\mathrm{R}}, P_{\mathrm{R}}$, and $t_{\mathrm{R}}$.

Once the material parameters are determined, it is then possible to predict the load-displacement-time response for an arbitrary load spectrum. Comparison of such predictions with measured responses is a test of the range of validity of the parameters and of the model. Here, for simplicity, the material parameters are used to predict the response to a linear load ramp to peak load $P_{\mathrm{U}}$ in time $t_{\mathrm{U}}$. The full displacement response is given by

$$
\begin{aligned}
h(t)=\left(P_{\mathrm{U}} t / t_{\mathrm{U}} \alpha_{1} H\right)^{1 / 2} & +\left(\frac{P_{\mathrm{U}}}{\alpha_{2} M_{1}}\right)^{1 / 2}\left[\left(t / t_{\mathrm{U}}\right)^{1 / 2}-\frac{\sqrt{\pi}}{2}\left(\tau_{1} / t_{\mathrm{U}}\right)^{1 / 2} \exp \left(-t / \tau_{1}\right) \operatorname{erf}\left(t / \tau_{1}\right)^{1 / 2}\right] \\
& +\left(\frac{P_{\mathrm{U}}}{\alpha_{2} M_{2}}\right)^{1 / 2}\left[\left(t / t_{\mathrm{U}}\right)^{1 / 2}-\frac{\sqrt{\pi}}{2}\left(\tau_{2} / t_{\mathrm{U}}\right)^{1 / 2} \exp \left(-t / \tau_{2}\right) \operatorname{erf}\left(t / \tau_{2}\right)^{1 / 2}\right] .
\end{aligned}
$$


In practice, it was more convenient to carry out the fitting and prediction in the normalized coordinates of $t / t_{\mathrm{R}}, h / h_{\mathrm{R}}$, and $P / P_{\mathrm{R}}$ referenced to the parameters characterizing the ramp at the beginning of the trapezoidal segment (iii). Equations (8)-(10) then took on simpler forms and the normalized loads and displacements were of order unity, greatly simplifying fitting. More importantly, on normalization $\alpha_{2} M_{1}$ and $\alpha_{2} M_{2}$ were then determined as explicit fitting parameters from Eqs. (8) and (9). This determination enabled predictions from Eq. (10) to be expressed entirely in terms of the dimensionless experimental ratios $t_{\mathrm{U}} / t_{\mathrm{R}}$ and $P_{\mathrm{U}} / P_{\mathrm{R}}$ without recourse to explicit specification of the geometry terms $\alpha_{1}$ and $\alpha_{2}$ and thus of the material properties $H, M_{1}$, and $M_{2}$.

\section{RESULTS}

\section{A. Model validation via single-point measurements}

Experimental displacement measurements for the PMMA1 material, such as shown in Figs. 2(b) and 2(c), gave parameters of $\alpha_{1} H=(33.3 \pm 0.4) \mathrm{GPa}$, $\alpha_{2} M_{1}=(13.4 \pm 0.1) \mathrm{GPa}, \alpha_{2} M_{2}=(470 \pm 8) \mathrm{GPa}$, $\tau_{1}=(23.0 \pm 0.4) \mathrm{s}$, and $\tau_{2}=(547 \pm 14) \mathrm{s}$, where the values represent the means and standard deviations of best fits to Eqs. (7)-(9) from four separate three-segment indentation experiments. Here and throughout, $\left(\tau_{1}, M_{1}\right)$ are taken to characterize the faster, short time constant viscoelastic deformation process and $\left(\tau_{2}, M_{2}\right)$ the slower, long time constant process. Figure 3 shows as symbols the load-displacement behavior of the PMMA1 material during linear load ramps to $100 \mathrm{mN}$ with rises times from $20 \mathrm{~s}$ to $2000 \mathrm{~s}$. (For clarity, as in Figs. 2, 4 and 5, the data are offset and not every measurement is shown.) Inspection of Fig. 3 shows that the displacement at

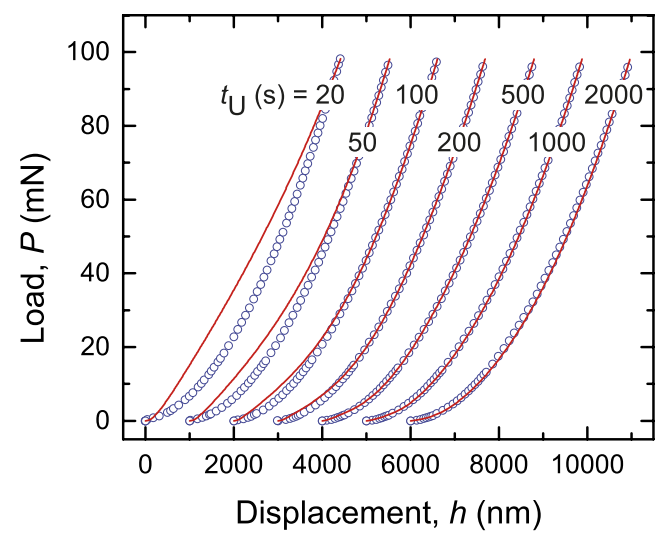

FIG. 3. Load-displacement responses of the PMMA1 material during linear load-time indentation ramps to the same peak load, but different rise times, $t_{\mathrm{U}}$. Symbols indicate experimental observations (not all shown) and the lines indicate predictions from measurements and analyses of three segment (Fig. 2) tests. Data offset horizontally for clarity. peak load increased from about $4000 \mathrm{~nm}$ for a rise time of $20 \mathrm{~s}$ to about $5000 \mathrm{~nm}$ for a rise time of $2000 \mathrm{~s}$, indicative of greater viscous flow as the test duration increased over this time scale. The solid lines in Fig. 3 are predictions of the load-displacement responses using Eq. (10) and the parameters given above. For rise times comparable to the creep hold time used to determine the viscoelastic parameters, $1000 \mathrm{~s}$, the predictions are a very good fit to the measurements. These fits reflect the fact that the $1000 \mathrm{~s}$ hold was easily able to capture the viscoelastic deformation processes associated with the longer $\tau_{2}$ time constant, $\approx 500 \mathrm{~s}$ [see Fig. 2(c)]. As the rise times decrease, the predictions do not fit the measurements as well, particularly in the early part of the experiments. These observations serve to place bounds on the validity of the model, consistent with the idea that events shorter than about two or three time constants for a modeled process will not be well described. In this case, the shorter $\tau_{1}$ time constant places an upper bound on the time scale for events to be well described of $\approx(40-60) \mathrm{s}$, in agreement with Fig. 3. The obvious remedy, at the cost of model complexity, is to increase the number of viscoelastic elements and time constants. ${ }^{16,18-20,38}$ As the inferred properties and resulting maps here used measurements deliberately not affected by the extreme short rise times used for illustration in Fig. 3, additional elements were not needed in this study.

Figure 4 shows as symbols the load-displacement behavior of the PMMA1 material during linear load ramps to $(20-500) \mathrm{mN}$ with a rise time of $500 \mathrm{~s}$. The solid lines in Fig. 4 are predictions of the loaddisplacement responses using Eq. (10) and the parameters given above. In distinction to Fig. 3, the predictions are a very good fit to the measurements for all the peak loads. This latter observation is

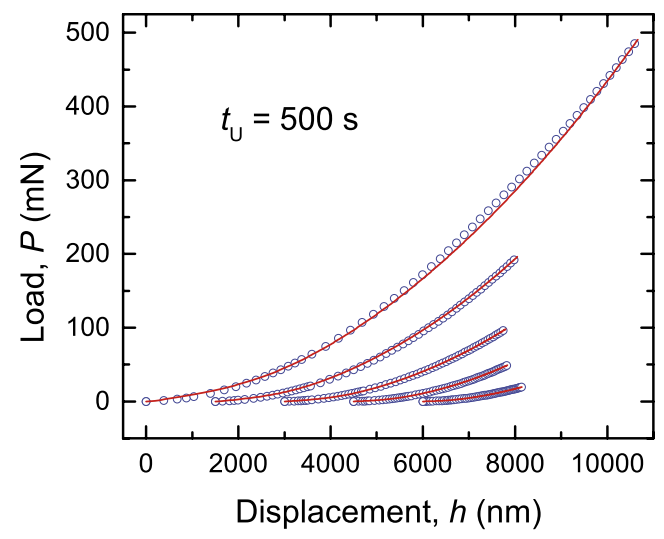

FIG. 4. Load-displacement responses of the PMMA1 material during linear load-time indentation ramps with the same rise time, but different peak loads. Symbols indicate experimental observations (not all shown) and the lines indicate predictions from measurements and analyses of three segment (Fig. 2) tests. Data offset horizontally for clarity. 
a consequence of the fact that the relative contributions to total deformation from plastic and viscoelastic processes remain fixed if the rise time is fixed even as the peak load (and hence loading rate) changes [Eqs. (9) and (10)]. Inserting $t=t_{\mathrm{U}}$ into Eq. (10) gives the displacement $h_{\mathrm{U}}$ at peak load of a ramp:

$$
\begin{aligned}
h_{\mathrm{U}}=\left(P_{\mathrm{U}} / \boldsymbol{\alpha}_{1} H\right)^{1 / 2} & +\left(P_{\mathrm{U}} / \boldsymbol{\alpha}_{2} M_{1}\right)^{1 / 2}\left[1-f\left(\tau_{1} / t_{\mathrm{U}}\right)\right] \\
& +\left(P_{\mathrm{U}} / \boldsymbol{\alpha}_{2} M_{2}\right)^{1 / 2}\left[1-f\left(\tau_{2} / t_{\mathrm{U}}\right)\right],
\end{aligned}
$$

where the functions $f$ are defined by Eq. (9) or (10) and depend only on the ratio of a material time constant and the experimental rise time. If the latter is fixed the values of the functions are fixed and hence the ratio of the first and second terms (the ratio of plastic and viscoelastic deformation) is also fixed. Equation (10a) also makes clear that load and loading rate also do not affect this ratio (providing material properties do not change with load). As the rise time used in Fig. 4 was fixed and long enough to capture the fast and slow viscoelastic processes, the relative contributions to total displacement and thus the shape of the load-displacement curves remained invariant; consistent with Eq. (10a), the predictions scaled simply with peak load over the load range used. (It is possible that plastic deformation will not be initiated or will be suppressed at very small loads, in which case $H$ would be load-dependent; this was not observed here.)

Figure 5 shows as symbols the load-displacement behavior of all the monolithic materials during linear load ramps with a rise time of $1000 \mathrm{~s}$. The solid lines in Fig. 5 are predictions of the load-displacement responses using Eq. (10) and the fitted mechanical deformation parameters, $\alpha_{1} H, \alpha_{2} M_{1}, \alpha_{2} M_{2}, \tau_{1}$, and $\tau_{2}$ for each material. In all cases, the predictions are very good fits to the

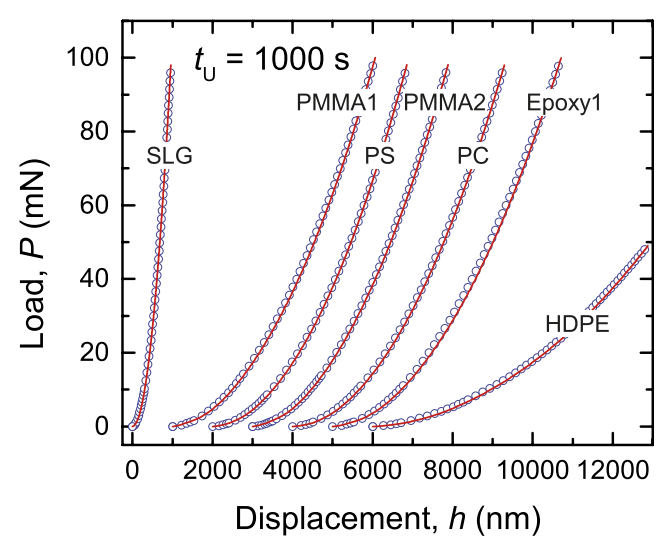

FIG. 5. Load-displacement responses of silicate glass and polymer materials during linear load-time indentation ramps. Symbols indicate experimental observations (not all shown) and the lines indicate predictions from measurements and analyses of three segment (Fig. 2) tests. Data offset horizontally for clarity. measurements. It is clear that SLG is much more resistant and HDPE much less resistant to indentation deformation under these conditions than the rest of the materials. It is also clear from the fits in Fig. 5 that the form of the analysis applies to a range of materials that exhibit viscoelastic and plastic deformation during pyramidal indentation. Comparison of the magnitudes of the fitted parameters $\alpha_{1} H$ and $\alpha_{2} M_{1}$ with commonly used values of hardness and Young's modulus ${ }^{39,40}$ for each material suggests that a very good approximation is that $\alpha_{1}$ and $\alpha_{2}$ are constants, and that the group of materials is well described by $\alpha_{1}=100$ and $\alpha_{2}=6$. Using these $\alpha$ parameters, Table I gives the values of $\tau_{1}, M_{1}, \tau_{2}, M_{2}$, and $H$ determined for each material, where the values represent the experimental means and standard deviations of best-fit parameters from four separate experiments. The $H$ values are comparable to those determined using quasi-static indentation ${ }^{40}$ and prior VEP methods. $^{1,2,4,7,9,20}$ The time constants are also comparable to those observed in indentation measurements in which a two time-constant model was used: a few tens of seconds for $\tau_{1}$ and a few hundreds of seconds for $\tau_{2}{ }^{3,9,18}$ The viscoelastic resistance parameters are comparable to those that can be inferred from a similar study, ${ }^{9}$ a few gigapascals for $M_{1}$ and a few tens of gigapascals for $M_{2}$, but the comparison is not direct as the prior study used a slightly different indentation model. A final point of comparison is that for SLG, which exhibited much greater resistance to viscoelastic deformation than the polymeric materials but similar time constants, in particular for the fast deformation process characterized by $\tau_{1}=11 \mathrm{~s}$. Such time dependence is consistent with earlier observations of hysteretic cyclic indentation of SLG at similar indentation time scales. ${ }^{41,42}$

\section{B. Viscoelastic and plastic property mapping}

Figure 6 shows property maps as color-fill contours over the same area for an extreme example of a change in properties at the Epoxy2-AlN interface; the interface is vertical and is located $400 \mu \mathrm{m}$ from the left edge of the images. To accommodate the extreme range of properties, Figs. 6(a)-6(c) use logarithmic contour intervals. Figure 6(a) is a map of the viscoelastic resistance parameter $M_{1}$. At this scale the epoxy appears uniform and is weakly resistant to viscoelastic deformation. The AlN ceramic is significantly more resistant to viscoelastic deformation and exhibits some variability in resistance (the weakening adjacent to the interface reflects the large contour intervals). Figure 6(b) is a similar map of the plastic resistance $H$; the appearance is similar to Fig. 6(a). In this extreme example of material changes, the interface between the epoxy and the ceramic appears sharp in both viscoelastic and plastic properties maps. Figure 6(c) is a map of the viscoelastic time constant $\tau_{1}$; 
TABLE I. Viscoelastic and plastic properties of materials.

\begin{tabular}{|c|c|c|c|c|c|}
\hline Material & $\begin{array}{l}\text { Viscoelastic time } \\
\text { constant, } \tau_{1}(\mathrm{~s})\end{array}$ & $\begin{array}{c}\text { Viscoelastic resistance, } \\
\qquad M_{1}(\mathrm{GPa})\end{array}$ & $\begin{array}{l}\text { Viscoelastic time } \\
\text { constant, } \tau_{2}(\mathrm{~s})\end{array}$ & $\begin{array}{c}\text { Viscoelastic resistance, } \\
\qquad M_{2}(\mathrm{GPa})\end{array}$ & $\begin{array}{c}\text { Plastic resistance, } \\
H(\mathrm{GPa})\end{array}$ \\
\hline HDPE & $26.3 \pm 0.5$ & $1.12 \pm 0.07$ & $494 \pm 8$ & $11.5 \pm 0.5$ & $0.052 \pm 0.004$ \\
\hline PMMA1 & $23.0 \pm 0.4$ & $2.23 \pm 0.02$ & $547 \pm 14$ & $78.3 \pm 1.3$ & $0.333 \pm 0.004$ \\
\hline PMMA2 & $21.3 \pm 0.1$ & $2.07 \pm 0.05$ & $499 \pm 10$ & $52.3 \pm 2.0$ & $0.330 \pm 0.010$ \\
\hline PS & $13.0 \pm 0.7$ & $4.0 \pm 0.2$ & $331 \pm 7$ & $262.5 \pm 7.5$ & $0.169 \pm 0.003$ \\
\hline PC & $9.2 \pm 0.4$ & $2.80 \pm 0.03$ & $310 \pm 18$ & $666.7 \pm 21.7$ & $0.148 \pm 0.009$ \\
\hline Epoxy1 & $18.9 \pm 0.4$ & $2.27 \pm 0.07$ & $481 \pm 10$ & $89.7 \pm 4.3$ & $0.171 \pm 0.007$ \\
\hline Epoxy2 & $19.8 \pm 0.4$ & $1.69 \pm 0.12$ & $560 \pm 20$ & $98.7 \pm 9.5$ & $0.273 \pm 0.015$ \\
\hline SLG & $11 \pm 2$ & $103 \pm 1$ & $1080 \pm 610$ & $(2.7 \pm 1.8) \times 10^{5}$ & $3.70 \pm 0.04$ \\
\hline
\end{tabular}

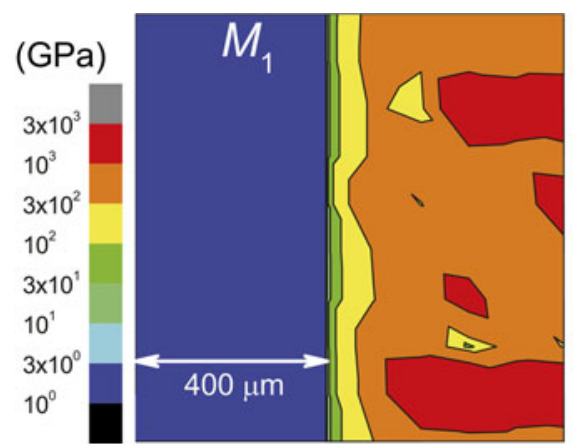

(a)

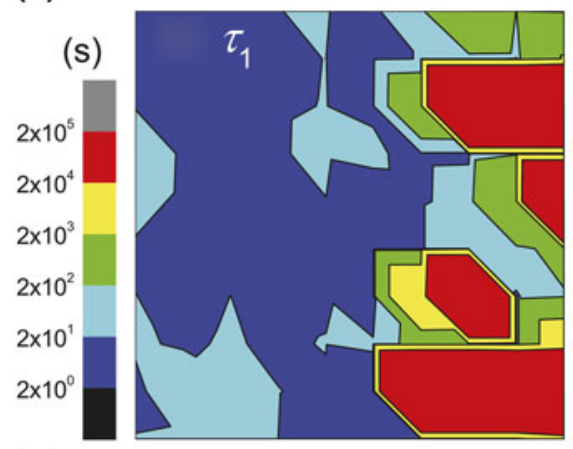

(c)

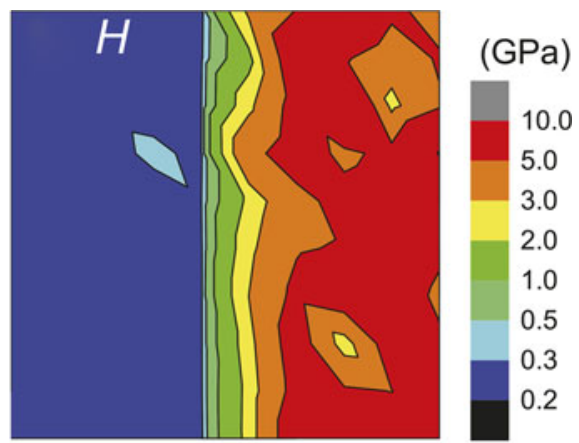

(b)

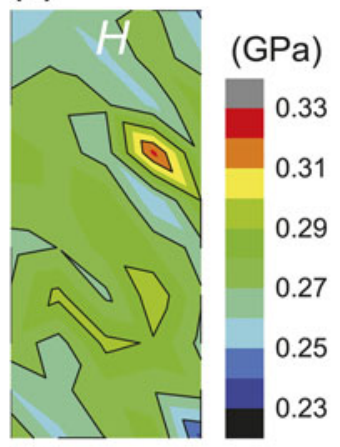

(d)

FIG. 6. Color-fill contour maps of mechanical properties variations at an epoxy-AlN interface; the epoxy extends $400 \mu \mathrm{m}$ from the left of the maps as indicated by the arrow. (a) The resistance to viscoelastic deformation, $M_{1}$, for the fast process in the epoxy. (b) The resistance to plastic deformation, $H$. (c) The viscoelastic deformation time constant, $\tau_{1}$, for the fast process. (d) The resistance to plastic deformation, $H$, in the epoxy, rescaled to enhance variation.

although the interface is less distinct, there is still a significant difference between the epoxy and the AlN, and there appears to be a correlation between $M_{1}$ and $\tau_{1}$ in the ceramic (in some regions there is more resistance to viscoelastic deformation and it is slower). Variability in the properties of the epoxy can be observed by changing the contour scale and this is shown in Fig. 6(d), which is a re-scaled map of $H$ for the epoxy alone. The epoxy plastic resistance varies by approximately $\pm 15 \%$ relatively but there does not seem to be an interface proximity effect as observed in the ceramic, Fig. 6(b).

Figures 7 and 8 show the variations in properties along line scans for the $1 \%$ and 5\% CNT-epoxy composites, respectively. The gray bands represent the mean \pm two standard deviation limits (given in Table I) of the properties determined for Epoxy1, the composite matrix. The symbols represent individual measurements in each of three separate scans; different symbols are used for each scan and the lines are guides to the eye. The composite materials exhibited both similarities and differences in comparison with the matrix material. For both composites, the time constants and resistances for the fast viscoelastic process, $\tau_{1}$ and $M_{1}$, top diagrams, were not significantly different from those of the matrix. For both composites, the time constants and resistances for the slow viscoelastic process, $\tau_{2}$ and $M_{2}$, center diagrams, were significantly greater than those of the matrix, particularly so for the slow viscoelastic resistance, $M_{2}$. 


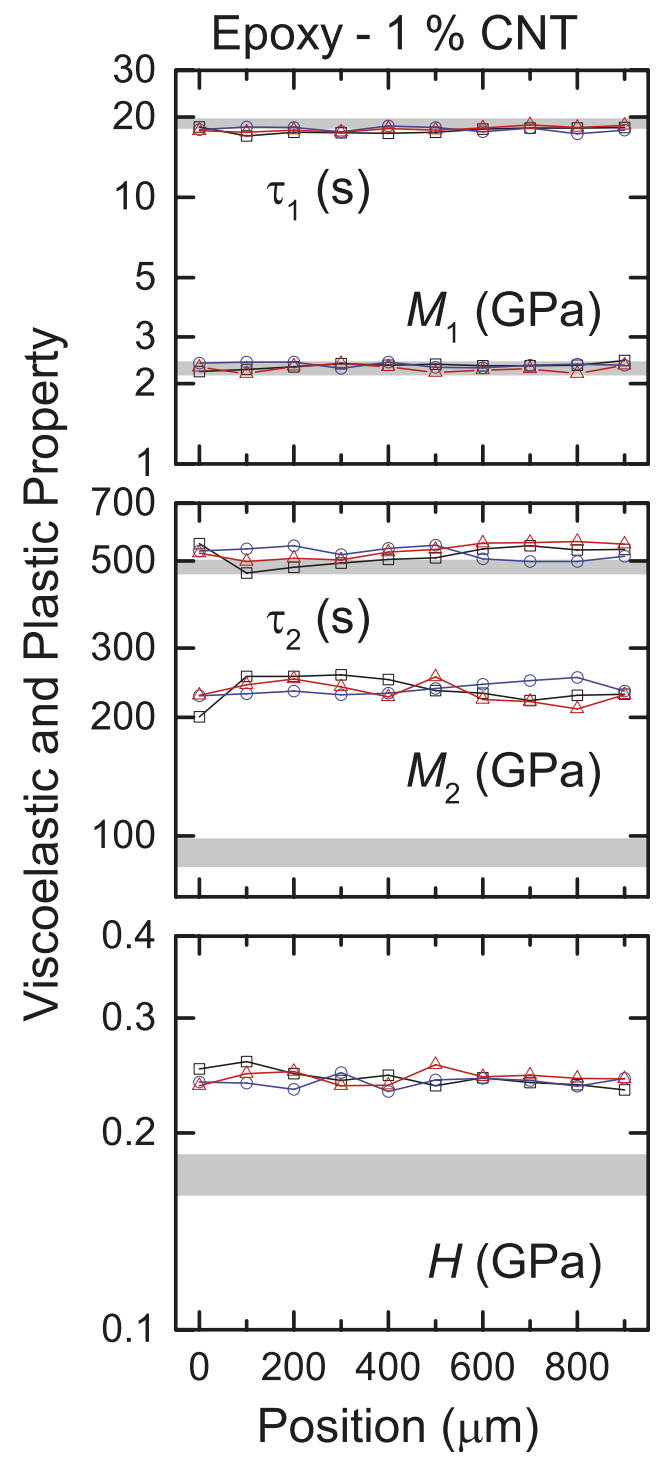

FIG. 7. Variations in mechanical properties of $1 \%$ CNT-epoxy composite along line scans. The gray bands indicate the properties of the epoxy matrix. (Upper) Time constant and resistance to deformation for the fast viscoelastic deformation process, $\left(\tau_{1}, M_{1}\right)$. (Central) Time constant and resistance to deformation for the slow viscoelastic deformation process, $\left(\tau_{2}, M_{2}\right)$. (Lower) Resistance to plastic deformation, $H$.

Similarly, for both composites, the resistances to plastic deformation, $H$, bottom diagrams, were significantly greater than that of the matrix. A significant difference between the composites was the variability in properties along the line scan. The 5\% composite exhibited much greater variability in all properties than the $1 \%$ composite, particularly so for $M_{2}$ and $H$.

Figures 9 and 10 show property maps for the $1 \%$ and 5\% CNT-epoxy composites, respectively. These maps provide pictorial illustrations of the above similarities and differences, as well as allowing an assessment of the composite microstructures and the characteristic length scales for variability or heterogeneity. For ease of

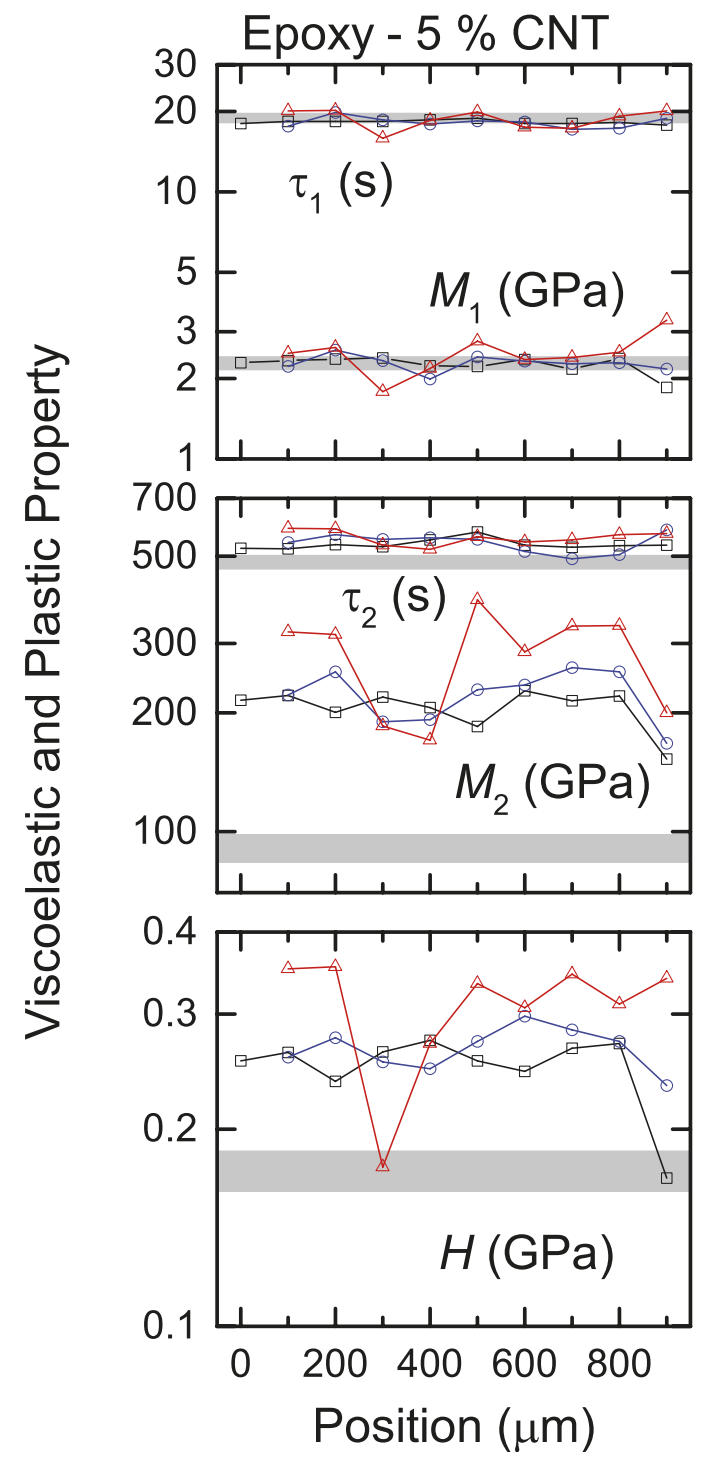

FIG. 8. Variations in mechanical properties of 5\% CNT-epoxy composite along line scans. Notation as in Fig. 7.

comparison, the maps are given as color-filled contours of relative properties, $X^{*}$ :

$$
X^{*}=\frac{X_{\text {composite }}-X_{\text {matrix }}}{X_{\text {matrix }}}
$$

such that $X^{*}=0$ corresponds to no difference from the matrix; the properties considered were $X=\tau_{2}, M_{2}$, and $H$ and the same contour intervals were used for each material. Figures 9(a) and 10(a) show maps of the relative time constants $\tau_{2}^{*}$ for the $1 \%$ and 5\% composites, respectively. In both cases, the "slow" process in the composite is even slower than that in the matrix $\left(\tau_{2}^{*}>0\right)$ and the variability and average of the time constant is greater in the 5\% material. Figures 9(b) and 10(b) show maps of the relative viscoelastic resistance $M_{2}^{*}$ for the $1 \%$ and $5 \%$ composites. In both cases, resistance to the slow deformation process is greater than that 


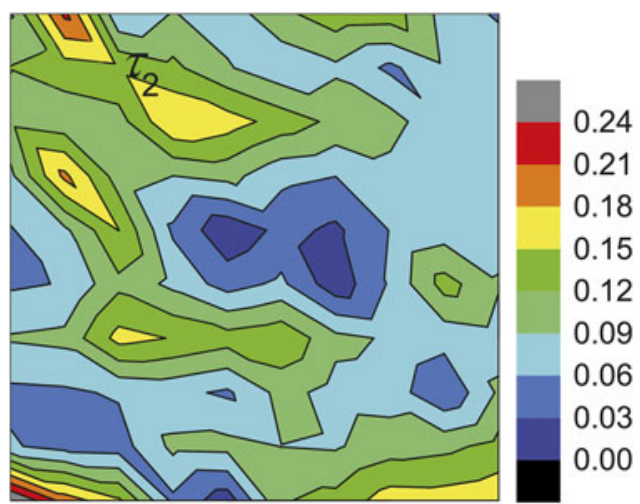

(a)

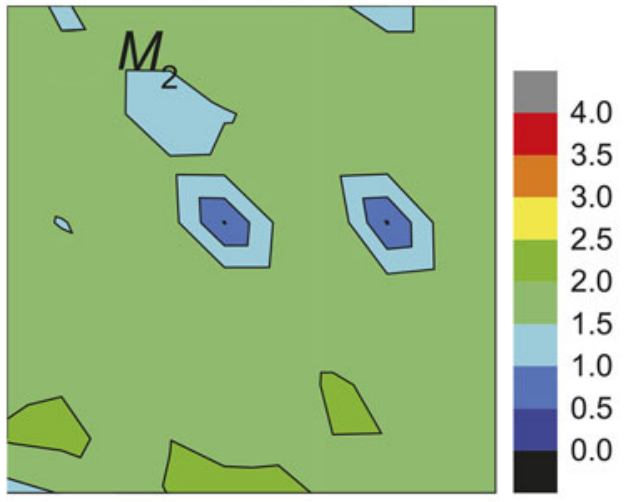

(b)

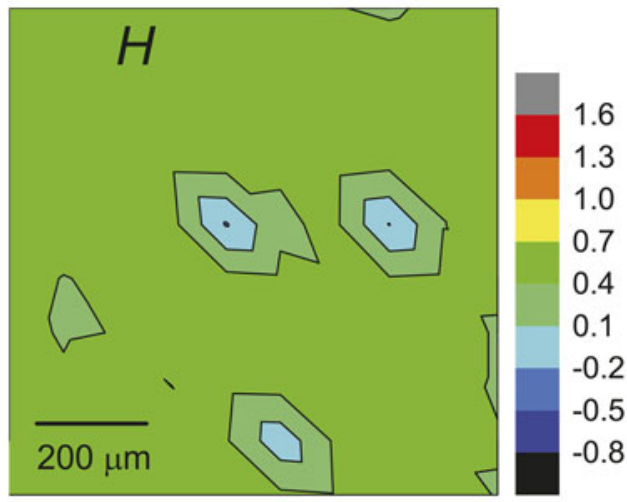

(c)

FIG. 9. Color-fill contour maps of mechanical properties variations in $1 \%$ CNT-epoxy composite. The properties are plotted relative to the epoxy matrix using Eq. (11). (a) The viscoelastic time constant, $\tau_{2}$, for the slow deformation process. (b) The resistance to viscoelastic deformation, $M_{2}$, for the slow process. (c) The resistance to plastic deformation, $H$.

in the matrix, particularly so for the $5 \%$ material. Finally, Figs. 9(c) and 10(c) show maps of the relative plastic resistance $H^{*}$ for the $1 \%$ and $5 \%$ composites. In this case, although the resistance to plastic deformation is mostly greater than that of the matrix, there are local "soft" spots $\left(H^{*}<0\right)$. The maps of Figs. 9 and 10 are of course consistent with the graphs of Figs. 7 and 8 with regard to values and variability, but they provide two additional

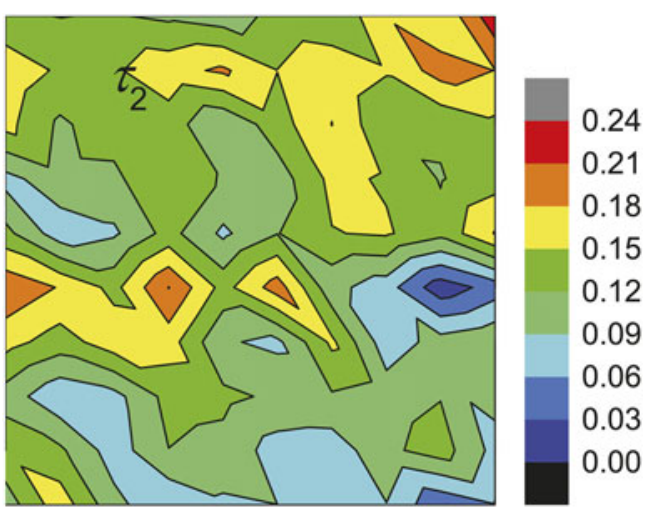

(a)

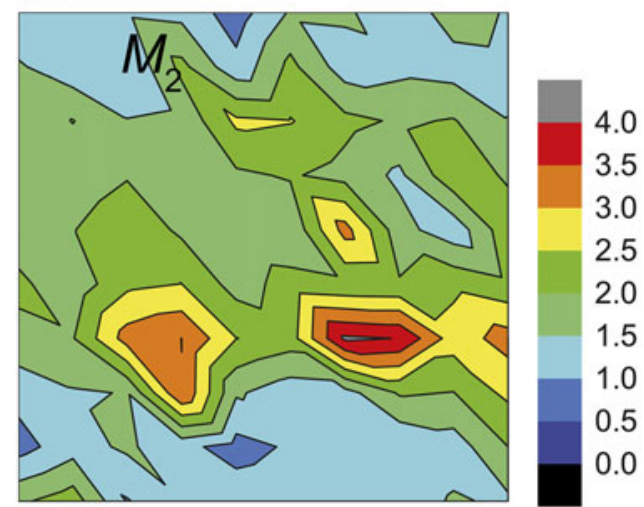

(b)

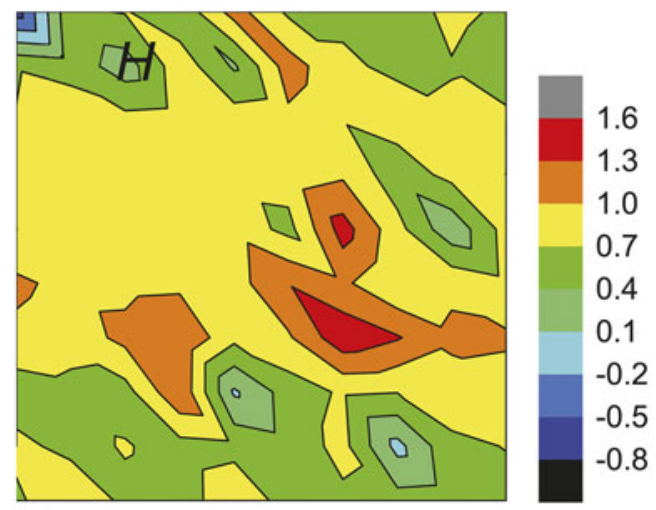

(c)

FIG. 10. Color-fill contour maps of mechanical properties variations in 5\% CNT-epoxy composite. Notation as in Fig. 9.

features. The first is that the form and length scales of the microstructures can easily be observed. The maps suggest that the microstructures are "clumped" with localized regions that are more resistant to deformation, and that these regions are approximately $200 \mu \mathrm{m}$ in size in $1 \%$ material and about $500 \mu \mathrm{m}$ in size in $5 \%$ material. The second is that correlations between properties can easily be observed. The maps suggest that there is a strong correlation between the resistances to viscoelastic and plastic deformation [maps (b) and (c)] and a weaker correlation between the viscoelastic time constant and resistance [maps (a) and (b)]. 


\section{DISCUSSION AND CONCLUSIONS}

The results presented here have demonstrated twodimensional mapping of viscoelastic and plastic properties of polymeric-based materials, extending the mapping capabilities to time-dependent deformation from those demonstrated previously ${ }^{32-37}$ using an elastic-plastic analysis. ${ }^{41}$ The previous studies all used indentation spacings smaller than the $100 \mu \mathrm{m}$ used here, ranging from $0.5 \mu \mathrm{m}^{32}-20 \mu \mathrm{m},{ }^{35}$ allowing most maps to be presented with properties as individual pixels ${ }^{32,34-36}$ rather than as contours ${ }^{33,37}$ as in Figs. 6, 9 and 10. In both the viscoelastic-plastic mapping here and in the previous elastic-plastic maps, the indentation spacing was matched to the scale of the microstructure and as a consequence there are many similarities between the previous maps and those obtained here: In previous maps of dense WC polycrystals, ${ }^{32}$ tooth enamel, ${ }^{33}$ lamellar bone, ${ }^{34}$ quartzite,${ }^{35}$ brass, ${ }^{36}$ and titanium, ${ }^{37}$ there were factors of two or three variation in modulus and hardness observed over the maps. These variations were associated with microstructural variations such as grain orientation or small variations in composition and are analogous to the variations in properties observed within the AlN ceramic and epoxy, Fig. 6. In some cases, much greater variations in properties were observed and were associated with abrupt changes in microstructure, such as decreases of factors of four in modulus and hardness associated with Co binder in WC-Co composites, ${ }^{32}$ decreases of factors of ten associated with graphite flakes in cast iron, ${ }^{36}$ and much greater decreases associated with porosity in bone $\mathrm{e}^{34}$ and cement. ${ }^{35}$ These latter variations are analogous to the variations in properties observed between the AlN and epoxy, Fig. 6.

The above observations and those of prior CNT-epoxy dispersion studies ${ }^{28,31}$ and reviews ${ }^{29,30}$ enable interpretation of the line scans and maps of Figs. 7-10 in terms of the CNT composite microstructures. Figures 7 and 8 show that in both composites the time constant and deformation resistance of the fast viscoelastic process $\left(\tau_{1}, M_{1}\right)$ are no different from that of the epoxy matrix. This observation suggests that the CNTs do not influence this deformation mechanism at all and that it is entirely associated with the epoxy and probably molecular in scale. Conversely, Figs. 7 and 8 show that for the slow viscoelastic process $\left(\tau_{2}, M_{2}\right)$ in both composites the time constant is increased somewhat and the deformation resistance is increased substantially from that of the epoxy. The implication here is that the incorporated CNTs are slowing this deformation process and making it more difficult, probably over length scales comparable to the indentation size, about $5 \mu \mathrm{m}$ (Fig. 5). Figures 7 and 8 also show that the resistance to plastic deformation, $H$, is increased substantially from that of the epoxy for both composites, consistent with the idea that the CNTs impeded both slow, time-dependent and irreversible, time-independent-deformation on length scales comparable to the indentation field. Figures 9 and 10 highlight, however, that the increases in $M_{2}$ and $H$ are not uniform: some areas exhibit less than average deformation resistance, e.g., in Fig. 9, and some areas exhibit greater than average deformation resistance, e.g., in Fig. 10, with strong correlation between $M_{2}$ and $H$. These areas probably reflect localized increased concentrations of CNTs and are comparable in size to the hundreds of micrometer- ${ }^{31}$ to millimeter-scale ${ }^{28}$ agglomerates observed previously. Removal of such entangled agglomerates is a major focus of the many methods ${ }^{29,30}$ used to disperse CNTs in polymer matrices, as the agglomerates are only weakly infiltrated by the polymer and thereby degrade the composite properties relative to those that might be achieved by well-dispersed CNTs bound to the matrix. This degradation is probably the case in Fig. 9, which shows local "soft" spots. Counter to this is Fig. 10, which shows local "hard" spots possibly reflecting enhanced areas of CNT concentration with adequate polymer infiltration. The advantage of maps such as Figs. 9 and 10 is that local variations in properties are assessed directly and do not have to be inferred from measurements of entire composite components. ${ }^{31}$ An implication from Figs. 7-10 is that the CNTs in the 5\% material were less uniformly dispersed than in the 1\% material. Imaging spectroscopy methods, e.g., Raman spectroscopy as applied to singlewall CNT composites, ${ }^{43}$ could possibly be used to directly assess CNT dispersion for comparison with the mechanical measurements.

Application of the method developed here to prediction of properties for a particular material requires a few additional steps. First, to establish accuracy (how close a measurement represents a true or known value), independent measurements of properties should be performed so as to fix the geometry terms $\alpha_{1}$ and $\alpha_{2}$ for the materials class under consideration. The material-invariant approximation used here returns reasonable property values (Sec. III.A and Table I) for a range of materials, but does not necessarily apply in detail to a specific material. Such independent measurements should be viscoelastic experiments, noting that the $M$ values used here are viscoelastic resistances and include elastic moduli as lower bounds. Second, to establish precision (how close a measurement represents a mean value) sufficient measurements should be performed to place statistical bounds on the determined $\tau_{1}, M_{1}, \tau_{2}, M_{2}$, and $H$ parameters. Table I suggests that four measurements provide sufficient precision for homogeneous materials at the indentation scale used here, but indentations at smaller scales, particularly in materials with heterogeneous microstructures, will lead to less precision (e.g., as in the elastic-plastic indentation studies above $\mathrm{e}^{32,36,37}$ ) and require greater numbers of indentations. This last point pertains particularly to composite materials: If globally averaged properties 
are required for a composite, say to predict the overall response of a component, sufficient numbers of indentations over a large enough area are required so as to assess the characteristic length scales of the microstructure. This in turn sets the "representative volume element" for the material that is the minimum component scale that can be regarded as possessing average properties. Third, to validate the assumed constitutive law and measured deformation parameters, predictions should be made and tested against loading protocols close to those expected in component operation, or at least different from those used to determine the parameters. Here, predictions from the three-segment measurements (Fig. 2) were tested against ramp loading protocols (Figs. 3-5) for which closed-form solutions [Eq. (10)] were developed. Similar validations are often performed, ${ }^{1,2,4,8,18-20}$ but often not, especially when viscoelastic correspondence methods are used to analyze measurements. 3,9,12,13,16,17 In this regard, it is to be noted that for nonsimple loading protocols or those with multiple stages, the integrand of the general displacement integral [Eq. (A5)] will usually not be too pathological and therefore amenable to straightforward numerical integration. It is also to be noted that the viscoelastic correspondence methods ${ }^{23-27}$ do not allow for unloading of the indenter (formally, they only allow monotonically increasing contact radius), which is not an inherent limitation of the VEP approach and which can be tested experimentally. ${ }^{4,9,12}$ Extension to arbitrary, multiple-stage loading protocols including unloading and numerical prediction of load-displacement-time responses will be the subject of future work.

Finally, practical considerations for indentation-based mapping of the mechanical properties of polymeric systems are that time will nearly always be an inherent part of the measurement procedure and that the indentations will invariably be large. Hence, maps that involve viscoelastic properties will always take longer to generate than those that only involve elastic-plastic properties, and maps of "soft" materials will always require indentation spacing greater than that of hard materials. As noted above, matching the indentation spacing and size to the length scale of the microstructure is important such that maps do not over- or under-sample. In polymeric composite systems with microstructural scales smaller than that examined here, finely spaced line scans about three indentation dimensions apart might provide a compromise between generating an accurate assessment of the heterogeneity of time-dependent mechanical responses and maintaining reasonable test durations.

\section{ACKNOWLEDGMENTS}

This research was performed while AJG was initially an undergraduate student volunteer researcher at NIST and subsequently a member of the NIST Summer
Undergraduate Research Fellowship (SURF) program. The authors thank APV Engineered Coatings and Arkema for assistance with sample preparation and Drs. Doug Smith and Brian Bush of NIST for experimental assistance. Certain commercial equipment, instruments or materials are identified in this document. Such identification does not imply recommendation or endorsement by the National Institute of Standards and Technology, nor does it imply that the products identified are necessarily the best available for the purpose.

\section{REFERENCES}

1. M.L. Oyen and R.F. Cook: Load-displacement behavior during sharp indentation of viscous-elastic-plastic materials. J. Mater. Res. 18, 139 (2003).

2. M.L. Oyen, R.F. Cook, J.A. Emerson, and N.R. Moody: Indentation responses of time-dependent films on stiff substrates. J. Mater. Res. 19, 2487 (2004).

3. C.Y. Zhang, Y.W. Zhang, K.Y. Zeng, and L. Shen: Nanoindentation of polymers with a sharp indenter. J. Mater. Res. 20, 1597 (2005).

4. R.F. Cook and M.L. Oyen: Nanoindentation behavior and mechanical properties measurement of polymeric materials. Int. J. Mater. Res. 98, 370 (2007).

5. M.L. Oyen and C-C. Ko: Examination of local variations in viscous, elastic, and plastic indentation responses in healing bone J. Mater. Sci.: Mater. Med. 18, 623 (2007).

6. M.L. Oyen and R.F. Cook: A practical guide for analysis of nanoindentation data. J. Mech. Behav. Biomed. Mater. 2, 396 (2009).

7. S.E. Olesiak, M.L. Oyen, and V.L. Ferguson: Viscous-elasticplastic behavior of bone using Berkovich nanoindentation. Mech. Time-Depend. Mater. 14, 111 (2010).

8. Y. Wang and I.K. Lloyd: Time-dependent nanoindentation behavior of high elastic modulus dental resin composites J. Mater. Res. 25, 529 (2010).

9. P-E. Mazeran, M. Beyaoui, M. Bigerelle, and M. Guigon: Determination of mechanical properties by nanoindentation in the case of viscous materials. Int. J. Mater. Res. 103, 715 (2012).

10. M. Sakai, S. Kawaguchi, and N. Hakiri: Contact-area-based FEA study on conical indentation problems for elastoplastic and viscoelastic-plastic bodies. J. Mater. Res. 27, 256 (2012).

11. N. Rodriguez-Florez, M.L. Oyen, and S.J. Shefelbine: Insight into differences in nanoindentation properties of bone. J. Mech. Behav Biomed. Mater. 18, 90 (2013).

12. S.J. Isaza, P-E. Mazeran, K. El Kirat, and M-C. Ho Ba Tho: Time-dependent mechanical properties of rat femoral cortical bone by nanoindentation: An age-related study. J. Mater. Res. 29, 1135 (2014).

13. L. Cheng, X. Xia, W. Yu, L.E. Scriven, and W.W. Gerberich: Flat-punch indentation of viscoelastic material. J. Polym. Sci., Part B: Polym. Phys. 38, 10 (2000).

14. M. Sakai and S. Shimizu: Indentation rheometry for glass-forming materials. J. Non-Cryst. Solids 282, 236 (2001).

15. M. Sakai: Time-dependent viscoelastic relation between load and penetration for an axisymmetric indenter. Philos. Mag. A 82, 1841 (2002).

16. S. Yang, Y-W. Zhang, and K. Zeng: Analysis of nanoindentation creep for polymeric materials. J. Appl. Phys. 95, 3655 (2004).

17. L. Cheng, X. Xia, L.E. Scriven, and W.W. Gerberich: Sphericaltip indentation of viscoelastic material. Mech. Mater. 37, 213 (2005).

18. M.L. Oyen: Spherical indentation creep following ramp loading. J. Mater. Res. 20, 2094 (2005). 
19. J.M. Mattice, A.G. Lau, M.L. Oyen, and R.W. Kent: Spherical indentation load-relaxation of soft biological tissues. J. Mater. Res. 21, 2003 (2006).

20. M.L. Oyen: Analytical techniques for indentation of viscoelastic materials. Philos. Mag. 86, 5625 (2006).

21. S. Shimizu, T. Yanagimoto, and M. Sakai: Pyramidal indentation load-depth curve of viscoelastic materials. J. Mater. Res. 14, 4075 (1999).

22. M. Sakai, S. Shimizu, N. Miyajima, Y. Tanabe, and E. Yasuda: Viscoelastic indentation of iodine-treated coal tar pitch. Carbon 39, 605 (2001)

23. E.H. Lee and J.R.M. Radok: The contact problem for viscoelastic bodies. J. Appl. Mech. 27, 438 (1960).

24. S.C. Hunter: The Hertz problem for a rigid spherical indenter and a viscoelastic half-space. J. Mech. Phys. Solids 8, 219 (1960).

25. G.A.C. Graham: The contact problem in the linear theory of viscoelasticity. Int. J. Eng. Sci. 3, 27 (1965).

26. W.H. Yang: The contact problem for viscoelastic bodies. J. Appl. Mech. 32, 395 (1966).

27. T.C.T. Ting: The contact stresses between a rigid indenter and a viscoelastic half-space. J. Appl. Mech. 33, 845 (1966).

28. J. Sandler, M.S.P. Shaffer, T. Prasse, W. Bauhofer, K. Schulte, and A.H. Windle: Development of a dispersion process for carbon nanotubes in an epoxy matrix and the resulting electrical properties. Polymer 40, 5967 (1999).

29. X-L. Xie, Y-W. Mai, and X-P. Zhou: Dispersion and alignment of carbon nanotubes in polymer matrix: A review. Mater. Sci. Eng., $R$ 49, 89 (2005).

30. P-C. Ma, N.A. Siddiqui, G. Marom, and J-K. Kim: Dispersion and functionalization of carbon nanotubes for polymer-based nanocomposites: A review. Composites, Part A 41, 1345 (2010).

31. A.K. Chakraborty, T. Plyhm, M. Barbezat, A. Necola, and G.P. Terrasi: Carbon nanotube (CNT)-epoxy nanocomposites: A systematic investigation of CNT dispersion. J. Nanopart. Res. 13, 6493 (2011).

\section{APPENDIX: VEP MODEL DEVELOPMENT}

The starting point for the quadratic viscoelastic Kelvin element is the linear Kelvin element load-displacement function: ${ }^{38}$

$$
P=k h+\eta \frac{\mathrm{d} h}{\mathrm{~d} t}
$$

where $k$ is interpreted as a stiffness, $\eta$ is interpreted as a viscosity, and the load is interpreted as the sum of the loads supported by an elastic element $(k)$ and a viscous element $(\eta)$ in parallel. Equation (A1) can be re-written as

$$
P=\eta\left(\frac{h}{\tau}+\frac{\mathrm{d} h}{\mathrm{~d} t}\right)
$$

where

$$
\tau=\frac{\eta}{k}
$$

is a characteristic time constant. By analogy with the quadratic viscous element described by Eq. (3), the quadratic viscoelastic Kelvin element is described by squaring the right side of Eq. (A2a),
32. H. Engqvist and U. Wiklund: Mapping of mechanical properties of WC-Co using nanoindentation. Tribol. Lett. 8, 147 (2000).

33. J.L. Cuy, A.B. Mann, K.J. Livi, M.F. Teaford, and T.P. Weihs: Nanoindentation mapping of the mechanical properties of human molar tooth enamel. Arch. Oral Biol. 47, 281 (2002).

34. H.S. Gupta, U. Stachewicz, W. Wagermaier, P. Roschger, H.D. Wagner, and P. Fratzl: Mechanical modulation at the lamellar level in osteonal bone. J. Mater. Res. 21, 1913 (2006).

35. W. Zhu, J.J. Hughes, N. Bicanic, and C.J. Pearce: Nanoindentation mapping of mechanical properties of cement paste and natural rocks. Mater. Charact. 58, 1189 (2007).

36. N.X. Randall, M. Vandamme, and F-J. Ulm: Nanoindentation analysis as a two-dimensional tool for mapping the mechanical properties of complex surfaces. J. Mater. Res. 24, 679 (2009).

37. D. Goldbaum, R.R. Chromik, N. Brodusch, and R. Gauvin: Microstructure and mechanical properties of Ti cold-spray splats determined by electron channeling contrast imaging and nanoindentation mapping. Microsc. Microanal. 21, 570 (2015).

38. W.N. Findley, J.S. Lai, and K. Onaran: Creep and Relaxation of Nonlinear Viscoelastic Materials (Dover Publications, Inc., New York, 1989).

39. W.D. Callister: Materials Science and Engineering (John Wiley \& Sons, Inc., New York, 1997).

40. CES Selector, version 4.5 (Granta Design Limited, Cambridge, 2004).

41. W.C. Oliver and G.M. Pharr: An improved technique for determining hardness and elastic modulus using load and displacement sensing indentation experiments. J. Mater. Res. 7, 1564 (1992).

42. J. Thurn and R.F. Cook: Indentation-induced deformation at ultramicroscopic and macroscopic contacts. J. Mater. Res. 19, 124 (2004).

43. F. Du, R.C. Scogna, W. Zhou, S. Brand, J.E. Fischer, and K.I. Winey: Nanotube networks in polymer Nanocomposites: Rheology and electrical conductivity. Macromolecules 37, 9048 (2004).

44. Wolfram Mathematica: http://integrals.wolfram.com (accessed August 14, 2014).

$$
P=\alpha_{2} M \tau^{2}\left(\frac{h}{\tau}+\frac{\mathrm{d} h}{\mathrm{~d} t}\right)^{2}
$$

where the term $\alpha_{2} M \tau^{2}$ is here an effective or lumped quadratic viscoelastic resistance. The first term in the parentheses in Eq. (A3) represents the elastic contribution to the resistance and the second term the viscous contribution. Inverting Eq. (A3) leads to a differential equation for the viscoelastic displacement resulting from an imposed load spectrum:

$$
\frac{\mathrm{d} h}{\mathrm{~d} t}+\frac{h}{\tau}=\frac{1}{\tau}\left(\frac{P(t)}{\alpha_{2} M}\right)^{1 / 2} .
$$

This is a differential equation of the form

$$
\dot{h}+h R=Q
$$

with $R$ and $Q$ defined by Eq. (A4) that can be solved with use of an integrating factor

$$
\rho=\exp \left(\int R \mathrm{~d} t\right)=\exp (t / \tau)
$$


with solution

$$
\rho h=\int \rho Q \mathrm{~d} t+C
$$

where $C$ is a constant of integration. Hence, the viscoelastic displacement can be written as

$$
h=e^{-t / \tau} \int \frac{e^{-t / \tau}}{\tau}\left[\frac{P(t)}{\alpha_{2} M}\right]^{1 / 2} \mathrm{~d} t+C e^{-t / \tau} .
$$

The time spectra of interest here are linear, of the form

$$
P(t)=A+B t
$$

In particular, for a ramp load from zero to a peak of $P_{\mathrm{R}}$ in a time of $t_{\mathrm{R}}, A=0$ and $B=P_{\mathrm{R}} / t_{\mathrm{R}}$ in Eq. (A6), and the solution to Eq. (A5) is, using Mathematica, ${ }^{44}$

$h=(b t)^{1 / 2}-\frac{\sqrt{\pi}}{2}(b t)^{1 / 2} \exp (-t / \tau) \operatorname{erfi}\left[(t / \tau)^{1 / 2}\right]+C e^{-t / \tau}$,

where

$$
b=\frac{P_{\mathrm{R}}}{t_{\mathrm{R}} \alpha_{2} M} .
$$

The function erfi(z) is the complex error function given by $\operatorname{erfi}(z)=-i \operatorname{erf}(z)$, where $\operatorname{erf}(z)$ is the general error function, $z$ is complex, and $i^{2}=-1$. It is easy to show that if the argument $z=x$ is real that $\operatorname{erfi}(x)=\operatorname{erf}(x)$. This is case here, Eq. (A7a), such that

$$
h=(b t)^{1 / 2}-\frac{\sqrt{\pi}}{2}(b t)^{1 / 2} \exp (-t / \tau) \operatorname{erf}(t / \tau)^{1 / 2}, t \leq t_{\mathrm{R}}
$$

using $h=0$ at $t=0$ to show that $C=0$, and where the notation for $\operatorname{erf}(x)^{1 / 2}$ in Eq. (A8) follows directly from Eq. (A7a). The first term on the right side of Eq. (A8) represents the displacement of a free elastic element and the second term represents the modification by the viscous element. Setting $t=t_{\mathrm{R}}$ in Eq. (A8) gives $h\left(t_{\mathrm{R}}\right)=h_{\mathrm{R}}$, the viscoelastic displacement at the peak of the ramp load.

For a load hold at the peak value, after a ramp, $A=P_{\mathrm{R}}$ and $B=0$ in Eq. (A6), and the solution to Eq. (A5) is, using separation of variables,

$$
h=h_{\mathrm{R}}+\left[a^{1 / 2}-h_{\mathrm{R}}\right]\left[1-\exp \left(-\frac{\left(t-t_{\mathrm{R}}\right)}{\tau}\right)\right], t \geq t_{\mathrm{R}},
$$

where

$$
a=\frac{P_{\mathrm{R}}}{\alpha_{2} M} .
$$

The term $h_{\mathrm{R}}$ appears in both additive and multiplicative roles on the right side of Eq. (A9a), modifying the creep response [Eq. (A9)] by displacement accrued during the prior ramp [Eq. (A8)]. In this sense, $h_{\mathrm{R}}$ acts as a "ramp correction factor" used earlier in a linear viscoelastic context. ${ }^{18,19}$ Eq. (A9) contains an essential result of the analysis: For $\left(t-t_{\mathrm{R}}\right)>>\tau$ the creep displacement approaches a bounded value of $h \rightarrow\left(P_{R} / \alpha_{2} M\right)^{1 / 2}$ in a nonlinear manner.

Alternatively, for a ramp unload from the peak of $P_{\mathrm{R}}$ to zero in a time of $t_{\mathrm{R}}, A=2 P_{\mathrm{R}}$ and $B=-P_{\mathrm{R}} / t_{\mathrm{R}}$ in Eq. (A6), and the solution to Eq. (A5) is, using Mathematica, ${ }^{44}$

$$
\begin{aligned}
h= & (a+b t)^{1 / 2}-\frac{\sqrt{\pi}}{2}(b \tau)^{1 / 2} \exp \left(\frac{a+b t}{b \tau}\right) \operatorname{erf}\left(\frac{a+b t}{b \tau}\right)^{1 / 2} \\
& +h_{\mathrm{F}} \exp \left(\frac{a+b t}{b \tau}\right), t_{\mathrm{R}} \leq t \leq 2 t_{\mathrm{R}} \quad,
\end{aligned}
$$

where now

$$
a=\frac{2 P_{\mathrm{R}}}{\alpha_{2} M}, b=-\frac{P_{\mathrm{R}}}{t_{\mathrm{R}} \alpha_{2} M} .
$$

Setting $t=2 t_{\mathrm{R}}$ in Eqs. (A10a) and (A10b) gives $h\left(2 t_{\mathrm{R}}\right)=h_{\mathrm{F}}$, the displacement at the end of the triangular load spectrum. Noting that both Eqs. (A8), (A10a) and (A10b) must pertain at $t=t_{\mathrm{R}}, h_{\mathrm{F}}$ can be determined in terms of $h_{\mathrm{R}}$ and thus in terms of test and material parameters.

Combining Eqs. (A8), (A10a) and (A10b) generates load-displacement responses that are indistinguishable from those observed in experiments and determined using the earlier Maxwell-like VEP model during triangle-wave loading. ${ }^{1,4}$ In particular, for slow tests the current model exhibits the initial negative unloading slope and unloading "nose." The explicit behavior described by Eqs. (A10a) and (A10b) will not be used here, but it is noted that in a zero-load recovery segment after a triangular load spectrum, such that $A=B=0$, the viscoelastic displacement is given by the last term in Eq. (A5) alone, such that

$$
h=h_{\mathrm{F}} \exp \left(-\frac{\left(t-2 t_{\mathrm{R}}\right)}{\tau}\right), t \geq 2 t_{\mathrm{R}} .
$$

Equation (A11) contains another essential result of the analysis: For $\left(t-2 t_{\mathrm{R}}\right)>>\tau$ in a recovery segment, the viscoelastic displacement $h \rightarrow 0$ in a nonlinear manner. 